
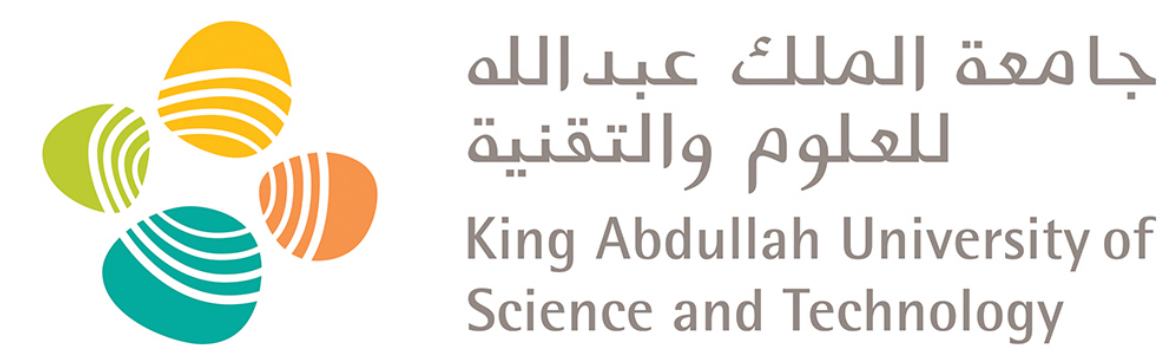

King Abdullah University of

Science and Technology

\title{
DC IR-Drop Analysis of Power Distribution Networks by a Robin Transmission Condition Enhanced Discontinuous Galerkin Method
}

\begin{tabular}{|c|c|}
\hline Item Type & Article \\
\hline Authors & $\begin{array}{l}\text { Yang, An Fa; Tang, Min; Mao, Jun Fa; Jiang, Li Jun; Bagci, Hakan; } \\
\text { Li, Ping }\end{array}$ \\
\hline Citation & $\begin{array}{l}\text { Yang, A. F., Tang, M., Mao, J. F., Jiang, L. J., Bagci, H., \& Li, P. } \\
\text { (2021). DC IR-Drop Analysis of Power Distribution Networks by a } \\
\text { Robin Transmission Condition Enhanced Discontinuous Galerkin } \\
\text { Method. IEEE Transactions on Components, Packaging and } \\
\text { Manufacturing Technology, 1-1. doi:10.1109/tcpmt.2021.3131513 }\end{array}$ \\
\hline Eprint version & Post-print \\
\hline $\mathrm{DOI}$ & 10.1109/ТСРМТ.2021.3131513 \\
\hline Publisher & IEEE \\
\hline Journal & $\begin{array}{l}\text { IEEE Transactions on Components, Packaging and Manufacturing } \\
\text { Technology }\end{array}$ \\
\hline Rights & $\begin{array}{l}\text { Archived with thanks to IEEE Transactions on Components, } \\
\text { Packaging and Manufacturing Technology }\end{array}$ \\
\hline Download date & 19/12/2021 10:53:51 \\
\hline Link to Item & http://hdl.handle.net/10754/673849 \\
\hline
\end{tabular}




\title{
DC IR-Drop Analysis of Power Distribution
}

\section{Networks by a Robin Transmission Condition Enhanced Discontinuous Galerkin Method}

\author{
An Fa Yang, Min Tang, Member, IEEE, Jun Fa Mao, Fellow, IEEE, \\ Li Jun Jiang, Fellow, IEEE, Hakan Bagci, Senior Member, IEEE, \\ and Ping Li, Senior Member, IEEE
}

\begin{abstract}
In this work, a novel Robin transmission condition (RTC) enhanced discontinuous Galerkin (DG) method is proposed for the DC IR-Drop analysis of power distribution networks with Joule Heating effects included. Unlike the conventional DG method, the proposed DG method straightforwardly applied to discretize the second-order spatial partial differential governing equations for the electrostatic potential $\Phi$ and the steady-state temperature $T$, respectively. The numerical flux in DG used to facilitate the information exchange among neighboring subdomains introduces two additional variables: the current density $\mathbf{J}$ for the electrical potential equation and the thermal flux $\mathbf{q}$ for the thermal equation. To solve them, at the interface of neighboring subdomains a RTC is presented as the second equation to establish another connection for solutions in neighboring subdomains. With this strategy, the number of degrees of freedom (DoF) involved in the proposed RTC-DG method is dramatically reduced compared
\end{abstract}

This work was supported by the National Key Research and Development Program of China under Grant 2020YFA0709800, in part by Shanghai Committee of Science and Technology under Grant 20501130500, in part by NSFC under Grant 62071290. (Corresponding author: Ping Li)

A. F. Yang, M. Tang, J. F. Mao, and P. Li are with the Key Laboratory of Ministry of Education of China for Research of Design and Electromagnetic Compatibility of High Speed Electronic Systems, Shanghai Jiao Tong University, Shanghai 200240, China (e-mail: ping.li@sjtu.edu.cn).

L. J. Jiang is with the Department of Electrical and Electronic Engineering, The University of Hong Kong, Pokfulam Road, Hong Kong.

H. Bagci is with the Division of Computer, Electrical, and Mathematical Sciences and Engineering and the Center for Uncertainty Quantification in Computational Science and Engineering, King Abdullah University of Science and Technology (KAUST), Thuwal, 23955-6900, Saudi Arabia. 
with the traditional DG method. The finalized matrix system is solved in a finite-element tearing and interconnecting (FETI)-like procedure, namely, the unknowns are obtained in a subdomain-by-subdomain scheme. Finally, the accuracy and the efficiency of the proposed RTC-DG method is validated by serval representative examples.

\section{Index Terms}

Domain decomposition method (DDM), discontinuous Galerkin (DG) method, DC IR-drop, electricalthermal co-simulation, Robin transmission condition (RTC).

\section{INTRODUCTION}

The reliability problems are becoming more serious with the continuous decreasing DC operation voltage, the increasing integration density, and the ever-shrinking feature sizes. Among them, DC IR-Drop [1] is a major issue in the power integrity (PI) problem, which would bring significant challenges to the design of the power distribution networks (PDN) of integrated circuits (IC) and printed circuit broads (PCB) [2]. In addition, Joule heating resulted thermal effects could be considerable with the shrinking scale of vias and traces, which would have serious impact on the resistivity of the conductors of PDNs. A comprehensive DC IR-drop analysis of PDNs should include the thermal effects. Otherwise, the voltage drop could be underestimated, which imposes a potential threat to the PI design. Thereby, the electrical-thermal co-simulation for DC IR-drop analysis is particularly necessary. For this purpose, extensive researches have been performed in the last few years.

Initially, the DC IR-Drop analysis is on the basis of the latency insertion method (LIM) [3] and the equivalent circuit model [4]. Soon after, the electrical-thermal co-simulation has been exploited with numerical algorithms such as the finite element method (FEM) [5]-[7] and the finite volume method (FVM) [8], which are capable of dealing with the complex geometries. As a integration of FEM and FVM, discontinuous Galerkin (DG) method [9], [10] absorbs the advantages of FVM and FEM, which provides a better candidate with respect to the accuracy and the parallel efficiency. For instance, to achieve high-order accuracy, like FEM [11], DG method is able to use the hierarchical high-order basis functions to approximate the solution. But More flexibly, the type and the order of basis functions in each element or subdomain are independent. On the other hand, same as FVM [12], DG method assumes that the solutions in each subdomain are individual to each other, and all information exchange among solutions in neighboring 
subdomains is facilitated via numerical flux, thus it is a natural domain decomposition method (DDM) and very suitable for parallel computing.

To leverage the domain decomposition capability of DG, in [13], a DG method is proposed for the transient thermal analysis of three-dimensional (3D) IC packages, where the transient thermal equation is solved in an element-by-element scheme with explicit time-marching strategy. With similar methodology, in [14], the DG method is extended to conduct the transient electromagnetic-thermal co-simulation for dispersive media. A further step is made in [15], where the DG method is applied to do steady-state electro-thermal co-analysis, where the Block Thomas method is employed to solve the original globally coupled matrix system in a subdomainby-subdomain scheme with Block Thomas method. Even though the capability of DG method in electrical-thermal analysis has been validated in these works, these DG based methods suffer from a common drawback, namely, the second-order governing partial differential equations (PDE) have to be degenerated to first-order ones. The additionally introduced variables (such as current density $\mathbf{J}=-\sigma \nabla \Phi$ and thermal flux $\mathbf{q}=-\kappa \nabla T$ ) in the degeneration process are present in the whole computational domain, thus the number of DoF is significantly increased, which is much larger compared with FEM.

With the aim to reduce the number of DoF suffered in the conventional DG method but simultaneously keep the domain decomposition capability of DG, in this work, a RTC enriched DG method is presented to conduct the DC IR-drop analysis of PDNs with thermal effects considered. Unlike the traditional DG method, the proposed DG method is applied directly to solve the second-order governing PDEs, namely, the Laplace equation and the Poisson equation respectively gauging the electrostatic potential and the steady-state thermal distributions. In DG method, the solutions in each subdomain are assumed to be independent with each other, to facilitate the information communication among solutions, usually a term called numerical flux is employed. For the electrostatic potential equation, the numerical flux is not only a function of potential $\Phi$, but also a function of current density $\mathbf{J}$; for the steady-state thermal equation, the numerical flux is not only a function of temperature $T$, but also a function of thermal flux q. Thus, to solve the additionally introduced unknowns, a Robin transmission condition (RTC) serving as the auxiliary equation is established at the interface of neighboring subdomains for the electrostatic potential equation and the steady-state thermal equation, respectively. In this way, the unknowns $\mathbf{J}$ and $\mathbf{q}$ are merely located at the interface of neighboring subdomains, thus they are surface unknowns. However, in traditional DG method, the second-order governing equations 
have to be degenerated to first-order ones, which results the additionally introduced unknowns $\mathbf{J}$ and $\mathbf{q}$ are present in the whole computational domain, namely, they are volume unknowns. Therefore, the proposed RTC-DG method in this work significantly decreases the number of DoF compared with the traditional DG.

Instead of solving a globally coupled matrix system, the finalized matrix system is solved in a FETI-like procedure [16]. The whole procedure involves two steps: firstly, the unknowns at the interfaces of neighboring subdomains are solved; then, the unknowns in the internal of each subdomain are solved simultaneously in a parallel way. In both steps, the sparse direct solver PARDISO [17] is employed to solve the corresponding matrix equations. Compared with the iterative methods such as the generalized minimum residual (GMRES) method, the deployed solving strategy is much more efficient and is also free of convergence issue.

The organization of the remainder of the paper is as follow. In Section II, the mathematical formulation of the proposed RTC-DG method for the electrostatic potential equation and the steady-state thermal equation is introduced, also the direct solver is described in detail. In Section III, the electrical-thermal cosimulation scheme is provided. In Section IV, several representative numerical examples are presented to verify the accuracy and convergence performance of the proposed method. Finally, the conclusion is summarized.

\section{FORMULATION}

Before the introduction of mathematical details of the proposed method, the computational domain of interesting (denoted as $\Omega$ ) is firstly split into $K$ non-overlapping subdomains (denoted as $\Omega^{k}$ ), and each subdomain is further decomposed into a number of tetrahedrons $\Omega_{k}^{i}$ $\left(i=1, \cdots, K_{k}\right)$, and then the DG formulation is applied to the governing equations in each subdomain.

\section{A. Formulation of Electrostatic Potential Equation by RTC-DG}

For DC IR-drop analysis, the electrostatic potential $\Phi$ is governed by the Laplace equation given by

$$
\nabla \cdot \sigma \nabla \Phi(\mathbf{r})=0
$$


which subjects to the boundary condition defined as

$$
\begin{aligned}
& \Phi=\Phi_{0} \quad \text { on } \Gamma_{\mathrm{dc}} \\
& -\sigma \frac{\partial \Phi}{\partial n}=\frac{\Phi}{R_{L} S} \text { on } \Gamma_{R}
\end{aligned}
$$

where $\sigma$ is the electrical conductivity of the conductors, $\Gamma_{\mathrm{dc}}$ is the Dirichlet boundary surface with potential already given, $\Gamma_{R}$ is the impedance boundary surface connecting with external load such as decoupling capacitors, match network, chips and memories, $R_{L}$ is the resistance value of the external equivalent load, and $S$ is the area of the cross section of the impedance boundary surface.

To establish the matrix equation in the $k$-th sudomain, the DG test is applied to (1), which leads to the following equation:

$$
\int_{\Omega_{k}} \nabla \psi_{i}^{k} \cdot \sigma^{k} \nabla \Phi^{k} \mathrm{~d} V-\int_{\partial \Omega_{k l}} \psi_{i}^{k}\left[\hat{\mathbf{n}}^{k l} \cdot\left(\sigma^{k} \nabla \Phi^{k}\right)\right] \mathrm{d} S=0
$$

where $\psi_{i}^{k}$ is the $i$-th testing function for potential $\Phi^{k}$ in subdomain $\Omega_{k}$, and $\hat{\mathbf{n}}^{k l}$ is an unit outward normal vector pointing from the current subdomain $\Omega_{k}$ to its neighbor $\Omega_{l}$ via the interface $\partial \Omega_{k l}$.

Due to the tangential continuity of solutions crossing the interface $\partial \Omega_{k l}$ in DG method is weakly enforced, numerical flux must be utilized to facilitate the information communication among adjacent subdomains. With the relation between the conductive current density and the electric field inside the conductor given by

$$
\mathbf{J}^{k}=-\sigma^{k} \nabla \Phi^{k}
$$

(2) is accordingly reformulated as

$$
\int_{\Omega_{k}} \nabla \psi_{i}^{k} \cdot \sigma^{k} \nabla \phi^{k} \mathrm{~d} V+\int_{\partial \Omega_{k l}} \psi_{i}^{k}\left(\hat{\mathbf{n}}^{k l} \cdot \mathbf{J}^{*}\right) \mathrm{d} S=0
$$

where $\hat{\mathbf{n}}^{k l} \cdot \mathbf{J}^{*}$ is the numerical flux, which is defined as

$$
\begin{aligned}
\hat{\mathbf{n}}^{k} \cdot \mathbf{J}^{*}= & C_{0}^{e}\left(\hat{\mathbf{n}}^{k l} \cdot \mathbf{J}^{k}-\hat{\mathbf{n}}^{l k} \cdot \mathbf{J}^{l}\right)+C_{1}^{e}\left(\hat{\mathbf{n}}^{k l} \cdot \mathbf{J}^{k}+\hat{\mathbf{n}}^{l k} \cdot \mathbf{J}^{l}\right) \\
& +C_{2}^{e}\left(\Phi^{k}-\Phi^{l}\right)
\end{aligned}
$$

with $\hat{\mathbf{n}}^{l k}=-\hat{\mathbf{n}}^{k l}$. In this work, the upwind flux is used, thus the corresponding parameter coefficients are defined by: $C_{0}^{e}=0, C_{1}^{e}=0.5$, and $C_{2}^{e}=-4$.

It is observed that the numerical flux in (5) is a function of both the electric potential $\Phi$ and the current density J. Therefore, in order to solve these two unknown variables together, one additional auxiliary equation is required. 
In the traditional DG methods such as the works in [13]-[15], the auxiliary equation is obtained via transforming the second-order PDE in (1) into two first-order PDEs, but which makes $\mathbf{J}$ as a volume unknown, i.e., it has to be solved in the whole computational domain. As a result, the number of DoFs increases dramatically, resulting in excessively computational cost.

Since the numerical flux in (5) merely happens at the interfaces of neighboring subdomains, thus $\mathbf{J}$ is a two-dimensional unknown variable that also only appears at the subdomain's interfaces. In this way, the auxiliary equation is desired to confine the $\mathbf{J}$ at the interfaces of neighboring subdomains instead of the entire computational domain. To reach this aim, a Robin transmission condition (RTC) that connects the solutions in current subdomain $k$ and its neighbor $l$ is established, which is derived on the basis of the following continuity relations:

$$
\Phi^{k}=\Phi^{l} \quad \text { on } \quad \partial \Omega_{k l}
$$

and

$$
\hat{\mathbf{n}}^{k l} \cdot \mathbf{J}^{k}=-\hat{\mathbf{n}}^{l k} \cdot \mathbf{J}^{l} \quad \text { on } \quad \partial \Omega_{k l}
$$

With (6) and (7), a general RTC can be obtained as

$$
\beta_{1}\left(\Phi^{k}-\Phi^{l}\right)+\beta_{2}\left(\hat{\mathbf{n}}^{k l} \cdot \mathbf{J}^{k}+\hat{\mathbf{n}}^{l k} \cdot \mathbf{J}^{l}\right)=0
$$

where two scaling factors $\beta_{1}$ and $\beta_{2}$ are introduced to balance the magnitude difference between $\Phi$ and $\mathbf{J}$ as well as to improve the condition number of later formed matrix equation. Refer to the relation in (3), it is assumed that $\beta_{1}=1$ and $\beta_{2}=\sigma^{k}$ in this wrok.

Next, the Galerkin test is applied to (8), which leads to the following formulation:

$$
\int_{\partial \Omega_{k l}} \varphi_{i}^{k} \cdot\left[\left(\Phi^{k}-\Phi^{l}\right)+\sigma^{k}\left(\mathrm{~J}_{n}^{k}+\mathrm{J}_{n}^{l}\right)\right] S=0
$$

with $\varphi_{i}^{k}$ denoting the $i$-th testing function, $\mathrm{J}_{n}^{k}=\hat{\mathbf{n}}^{k l} \cdot \mathbf{J}^{k}$ and $\mathrm{J}_{n}^{l}=\hat{\mathbf{n}}^{l k} \cdot \mathbf{J}^{l}$ indicating the normal component of $\mathbf{J}^{k / l}$, respectively.

Then, to establish a fully-discrete matrix system, $\Phi^{k / l}$ and $J_{n}^{k / l}$ in (4) and (9) are further approximated by nodal basis functions:

$$
\begin{aligned}
\Phi^{k / l} & =\sum_{j=1}^{n_{\Phi}^{k / l}} \gamma_{j}^{k / l} \phi_{j}^{k / l} \\
\mathrm{~J}_{n}^{k / l} & =\sigma \sum_{j=1}^{n_{\mathrm{J}}^{k / l}} \lambda_{j}^{k / l} \varphi_{j}^{k / l}
\end{aligned}
$$


where $n_{\Phi}^{k / l}$ and $n_{\mathrm{J}}^{k / l}$ denote the total number of the basis functions for $\Phi^{k / l}$ and $\mathrm{J}^{k / l}$ in the $k$ and $l$-th subdomains, respectively; $\gamma_{j}^{k / l}$ and $\lambda_{j}^{k / l}$ are the corresponding expansion coefficients to be determined.

With (5), (10), and (11), a compact form of (4) and (9) can be obtained as

$$
\left[\begin{array}{cc}
\mathbf{T}_{e}^{k} & \mathbf{0} \\
\mathbf{0} & \mathbf{0}
\end{array}\right]\left[\begin{array}{c}
\gamma^{k} \\
\lambda^{k}
\end{array}\right]+\sum_{l=1}^{K}\left[\begin{array}{cc}
\mathbf{F}_{\Phi \Phi}^{k l} & \mathbf{F}_{\Phi \mathrm{J}}^{k l} \\
\mathbf{F}_{\mathrm{J} \Phi}^{k l} & \mathbf{F}_{\mathrm{JJ}}^{k l}
\end{array}\right]\left[\begin{array}{c}
\gamma^{l} \\
\lambda^{l}
\end{array}\right]=\left[\begin{array}{c}
\boldsymbol{f}_{e}^{k} \\
\mathbf{0}
\end{array}\right]
$$

where $\boldsymbol{f}_{e}^{k}$ is a column vector rising from the Dirichlet boundary condition; $\mathbf{F}_{\Phi \Phi}^{k l}, \mathbf{F}_{\Phi \mathrm{J}}^{k l}, \mathbf{F}_{\mathrm{J} \Phi}^{k l}$, and $\mathbf{F}_{\mathrm{JJ}}^{k l}$ denote the coupling matrices between the $k$-th subdomain and the $l$-th subdomain, respectively. It is necessary to remind that the coupling matrices are zero if the $l$-th subdomain is not the neighbor of the $k$-th subdomain. In the following, the definitions of matrix elements are given by

$$
\begin{aligned}
& {\left[\mathbf{T}_{e}^{k}\right]^{i j}=\int_{\Omega_{k}} \nabla \phi_{i}^{k} \cdot \sigma^{k} \nabla \phi_{j}^{k} \mathrm{~d} V} \\
& {\left[\mathbf{F}_{\Phi \Phi}^{k k}\right]^{i j}=-4.0 \int_{\partial \Omega_{k}} \phi_{i}^{k} \cdot \phi_{j}^{k} \mathrm{~d} S} \\
& {\left[\mathbf{F}_{\Phi \Phi}^{k l}\right]^{i j}=4.0 \int_{\partial \Omega_{k l}} \phi_{i}^{k} \cdot \phi_{j}^{l} \mathrm{~d} S} \\
& {\left[\mathbf{F}_{\Phi \mathrm{J}}^{k k}\right]^{i j}=0.5 \sigma^{k} \int_{\partial \Omega_{k}} \phi_{i}^{k} \cdot \varphi_{j}^{k} \mathrm{~d} S} \\
& {\left[\mathbf{F}_{\Phi \mathrm{J}}^{k l}\right]^{i j}=-0.5 \sigma^{k} \int_{\partial \Omega_{k l}} \phi_{i}^{k} \cdot \varphi_{j}^{l} \mathrm{~d} S} \\
& {\left[\mathbf{F}_{\mathrm{J} \Phi}^{k k}\right]^{i j}=\sigma^{k} \int_{\partial \Omega_{k}} \varphi_{i}^{k} \cdot \phi_{j}^{k} \mathrm{~d} S} \\
& {\left[\mathbf{F}_{\mathrm{J} \Phi}^{k l}\right]^{i j}=-\sigma^{k} \int_{\partial \Omega_{k l}} \varphi_{i}^{k} \cdot \phi_{j}^{l} \mathrm{~d} S} \\
& {\left[\mathbf{F}_{\mathrm{JJ}}^{k k}\right]^{i j}=\sigma^{k} \int_{\partial \Omega_{k}} \varphi_{i}^{k} \cdot \varphi_{j}^{k} \mathrm{~d} S} \\
& {\left[\mathbf{F}_{\mathrm{JJ}}^{k l}\right]^{i j}=-\sigma^{k} \int_{\partial \Omega_{k l}} \varphi_{i}^{k} \cdot \varphi_{j}^{l} \mathrm{~d} S}
\end{aligned}
$$

Finally, by coupling the individual matrix in $K$ different subdomains together, a global matrix 
system can be obtained as

$$
\left[\begin{array}{ccccc}
\mathbf{M}_{e}^{1} & \mathbf{C}_{e}^{12} & \mathbf{C}_{e}^{13} & \cdots & \mathbf{C}_{e}^{1 K} \\
\mathbf{C}_{e}^{21} & \mathbf{M}_{e}^{2} & \mathbf{C}_{e}^{23} & \ldots & \mathbf{C}_{e}^{2 K} \\
\mathbf{C}_{e}^{31} & \mathbf{C}_{e}^{32} & \mathbf{M}_{e}^{3} & \ldots & \mathbf{C}_{e}^{3 K} \\
\vdots & \vdots & \vdots & \ddots & \vdots \\
\mathbf{C}_{e}^{K 1} & \mathbf{C}_{e}^{K 2} & \cdots & \mathbf{C}_{e}^{K, K-1} & \mathbf{M}_{e}^{K}
\end{array}\right]\left[\begin{array}{c}
\boldsymbol{v}_{e}^{1} \\
\boldsymbol{v}_{e}^{2} \\
\boldsymbol{v}_{e}^{3} \\
\vdots \\
\boldsymbol{v}_{e}^{K}
\end{array}\right]=\left[\begin{array}{c}
\tilde{f}_{e}^{1} \\
\tilde{\boldsymbol{f}}_{e}^{2} \\
\tilde{\boldsymbol{f}}_{e}^{3} \\
\vdots \\
\tilde{\boldsymbol{f}}_{e}^{K}
\end{array}\right]
$$

where $\boldsymbol{v}_{e}^{k}=\left[\gamma^{k}, \lambda^{k}\right]^{T}, \tilde{f}_{e}^{k}=\left[\boldsymbol{f}_{e}^{k}, \mathbf{0}\right]^{T}$, and

$$
\mathbf{M}_{e}^{k}=\left[\begin{array}{cc}
\mathbf{T}_{e}^{k}+\mathbf{F}_{\Phi \Phi}^{k k} & \mathbf{F}_{\Phi \mathrm{J}}^{k k} \\
\mathbf{F}_{\mathrm{J} \Phi}^{k k} & \mathbf{F}_{\mathrm{JJ}}^{k k}
\end{array}\right]
$$

and

$$
\mathbf{C}_{e}^{k l}=\left[\begin{array}{cc}
\mathbf{F}_{\Phi \Phi}^{k l} & \mathbf{F}_{\Phi \mathrm{J}}^{k l} \\
\mathbf{F}_{\mathrm{J} \Phi}^{k l} & \mathbf{F}_{\mathrm{JJ}}^{k l}
\end{array}\right]
$$

\section{B. Formulation of Steady-State Thermal Equation by RTC-DG}

For the steady-state thermal analysis, the heat equation is governed by

$$
\nabla \cdot \kappa \nabla T(\mathbf{r})=-Q(\mathbf{r})
$$

which is subject to the boundary conditions:

$$
\begin{gathered}
T=T_{0} \quad \text { on } \Gamma_{t c} \\
\kappa \frac{\partial T}{\partial n}=-h\left(T-T_{a}\right) \quad \text { on } \Gamma_{c o n v}
\end{gathered}
$$

with $\kappa$ indicating the thermal conductivity, $h$ designating the convection coefficient, and $Q$ representing the Joule heating source.

Similar to matrix formulation procedure of the electrical problem, the DG test is directly applied to (25) in the $k$-th subdomain,

$$
\int_{\Omega_{k}} \nabla \psi_{p}^{k} \cdot \kappa^{k} \nabla T^{k} \mathrm{~d} V-\int_{\partial \Omega_{k l}} \psi_{p}^{k}\left[\hat{\mathbf{n}}^{k} \cdot\left(\kappa^{k} \nabla T^{k}\right)\right] \mathrm{d} S=\int_{\Omega_{k}} \psi_{p}^{k} \cdot Q^{k} d V
$$

where $\psi_{p}^{k}$ is the $p$-th nodal basis function for the temperature distribution $T$ in the $k$-th subdomain.

By further resorting to the definition of thermal flux given as

$$
\mathbf{q}^{k}=-\kappa^{k} \nabla T^{k}
$$


then (26) can be rewritten as

$$
\int_{\Omega_{k}} \nabla \psi_{p}^{k} \cdot \kappa^{k} \nabla T^{k} \mathrm{~d} V-\int_{\partial \Omega_{k}} \psi_{p}^{k}\left[\hat{\mathbf{n}}^{k l} \cdot \mathbf{q}^{*}\right] \mathrm{d} S=\int_{\Omega_{k}} \psi_{p}^{k} \cdot Q^{k} d V
$$

where $\hat{\mathbf{n}}^{k l}$ is an unit outward normal vector pointing from the $k$-th subdomain to the $l$-th subdomain, and the term $\hat{\mathbf{n}}^{k l} \cdot \mathbf{q}^{*}$ is the numerical flux defined by

$$
\begin{aligned}
\hat{\mathbf{n}}^{k l} \cdot \mathbf{q}^{k}= & C_{0}^{h}\left(\hat{\mathbf{n}}^{k l} \cdot \mathbf{q}^{k}+\hat{\mathbf{n}}^{k l} \cdot \mathbf{q}^{l}\right)+C_{1}^{h}\left(\hat{\mathbf{n}}^{k l} \cdot \mathbf{q}^{k}-\hat{\mathbf{n}}^{k l} \cdot \mathbf{q}^{l}\right) \\
& +C_{2}^{h}\left(T^{k}-T^{l}\right)
\end{aligned}
$$

In this work, the upwind flux is employed, where the coefficients are defined as $C_{0}^{h}=0$, $C_{1}^{h}=0.5$ and $C_{2}^{h}=-4$.

It is noted that the numerical flux introduces new variable named as thermal flux q, thus to solve it, another auxiliary equation is needed. In traditional DG method, the PDE defined as $\nabla \cdot \mathbf{q}=Q$ is utilized as the auxiliary equation [13]-[15]. As a consequence, the unknown $\mathbf{q}$ has to be evaluated in the entire computational region, which no doubt increases the computational burden considerably.

In this work, in order to not only solve the new unknown q but also confine it at the interfaces of neighbor subdomains, a RTC is designed on the basis of the continuity of $T$ and $\hat{\mathbf{n}} \cdot \mathbf{q}$ across the interface $\partial \Omega_{k l}$, which is given by

$$
\tau_{1}\left(T^{k}-T^{l}\right)+\tau_{2}\left(q^{k}+q^{l}\right)=0
$$

where $q^{k}=\hat{\mathbf{n}}^{k l} \cdot \mathbf{q}^{k}$ and $q^{l}=\hat{\mathbf{n}}^{l k} \cdot \mathbf{q}^{l} ; \tau_{1}$ and $\tau_{2}$ are two factors used to make $T$ and $q$ at the same scale. In this work, $\tau_{1}=1$ and $\tau_{2}=\kappa^{k}$ are made on the basis of relation in (27).

Next, the Galerkin test is applied to (30) with trial function $\psi_{q}^{k}$

$$
\int_{\partial \Omega_{k l}} \psi_{q}^{k} \cdot\left[\left(T^{k}-T^{l}\right)+\kappa^{k}\left(q^{k}+q^{l}\right)\right] d S=0
$$

By further expanding the unknowns with nodal basis functions, namely,

$$
\begin{aligned}
T^{k / l} & =\sum_{j=1}^{n_{T}^{k / l}} \Lambda_{j}^{k / l} \nu_{j}^{k / l} \\
\mathrm{q}^{k / l} & =\sum_{j=1}^{n_{\mathrm{q}}^{k / l}} \Upsilon_{j}^{k / l} \psi_{j}^{k / l}
\end{aligned}
$$


with $n_{T}^{k / l}$ and $n_{\mathrm{q}}^{k / l}$ denoting the total number of basis functions for $T^{k / l}$ and $\mathrm{q}^{k / l}$, respectively; $\nu_{j}^{k / l}$ and $\psi_{j}^{k / l}$ representing the $j$-th scalar basis functions for $T^{k / l}$ and $\mathrm{q}^{k / l}$, respectively; $\Lambda_{j}^{k / l}$ and $\Upsilon_{j}^{k / l}$ indicating the corresponding expansion coefficients to be solved.

Then, with (29), (32) and (33), a compact form of (28) and (31) can be obtained as

$$
\left[\begin{array}{cc}
\mathbf{T}_{h}^{k} & \mathbf{0} \\
\mathbf{0} & \mathbf{0}
\end{array}\right]\left[\begin{array}{c}
\Lambda^{k} \\
\Upsilon^{k}
\end{array}\right]+\sum_{l=1}^{K}\left[\begin{array}{cc}
\mathrm{F}_{T T}^{k l} & \mathrm{~F}_{T \mathrm{q}}^{k l} \\
\mathrm{~F}_{\mathrm{q} T}^{k l} & \mathbf{F}_{\mathrm{qq}}^{k l}
\end{array}\right]\left[\begin{array}{c}
\Lambda^{l} \\
\Upsilon^{l}
\end{array}\right]=\left[\begin{array}{c}
\boldsymbol{f}_{h}^{k} \\
\mathbf{0}
\end{array}\right]
$$

Finally, the total $K$ subdomain matrix equations will couple together to form a global matrix system given as

$$
\left[\begin{array}{ccccc}
\mathbf{M}_{h}^{1} & \mathbf{C}_{h}^{12} & \mathbf{C}_{h}^{13} & \ldots & \mathbf{C}_{h}^{1 K} \\
\mathbf{C}_{h}^{21} & \mathbf{M}_{h}^{2} & \mathbf{C}_{h}^{23} & \ldots & \mathbf{C}_{h}^{2 K} \\
\mathbf{C}_{h}^{31} & \mathbf{C}_{h}^{32} & \mathbf{M}_{h}^{3} & \ldots & \mathbf{C}_{h}^{3 K} \\
\vdots & \vdots & \vdots & \ddots & \vdots \\
\mathbf{C}_{h}^{K 1} & \mathbf{C}_{h}^{K 2} & \ldots & \mathbf{C}_{h}^{K, K-1} & \mathbf{M}_{h}^{K}
\end{array}\right]\left[\begin{array}{c}
\boldsymbol{v}_{h}^{1} \\
\boldsymbol{v}_{h}^{2} \\
\boldsymbol{v}_{h}^{3} \\
\vdots \\
\boldsymbol{v}_{h}^{K}
\end{array}\right]=\left[\begin{array}{c}
\tilde{\boldsymbol{f}}_{h}^{1} \\
\tilde{\boldsymbol{f}}_{h}^{2} \\
\tilde{\boldsymbol{f}}_{h}^{3} \\
\vdots \\
\tilde{\boldsymbol{f}}_{h}^{K}
\end{array}\right]
$$

It is essential to explain that the detailed mathematical expressions of the matrix blocks in (34) and (35), as well as the associated matrix elements will not be shown here due to the limited space, but which can be readily derived by just alternating the basis functions and the material parameters in terms of the expressions given from (15) to (24).

\section{Comparison with the Traditional DG Method}

For electrical-thermal DC IR-drop analysis, the traditional DG method needs to degenerate the second-order PDEs in (1) and (25) into two first-order ones, and then DG test is applied to the two first-order PDEs. To have a more insightful understanding, the electrostatic potential governing equation is taken as example for illustration.

The traditional DG method [13]-[15] solves (1) by introducing an intermediate variable $\mathbf{J}$, then (1) is able to be reduced into two first-order PDEs defined by

$$
\begin{aligned}
& \mathbf{J}=-\sigma \nabla \Phi \\
& \nabla \cdot \mathbf{J}=0
\end{aligned}
$$

It is obviously noticed that the auxiliary variable $\mathbf{J}$ needs to be solved in the entire computational domain, which is particularly unfavorable with regarding to the computational performance.

Nevertheless, in this work, the proposed DG method directly discretizes the second-order governing PDEs in (1) and (25), and the RTC is exploited as the auxiliary equation. The most 
TABLE I

Number of DoFs for $\Phi$ And J With $N_{\Phi}, N_{\mathrm{J}}, N_{T}$, ANd $N_{\mathrm{q}}$ DENoting The number of DoFs For the Potential $\Phi$, THE CURRENT DENSITYJ, THE TEMPERATURE $T$, AND THERMAL FLUX q, RESPECTIVELY.

\begin{tabular}{|c|c|c|c|c|}
\hline Method & $N_{\Phi}$ & $N_{\mathrm{J}}$ & $N_{T}$ & $N_{\mathrm{q}}$ \\
\hline RTC-DG & 66,033 & 10,659 & 66,189 & 10,659 \\
\hline Traditional DG [15] & 66,033 & 198,099 & 66,189 & 10,659 \\
\hline
\end{tabular}

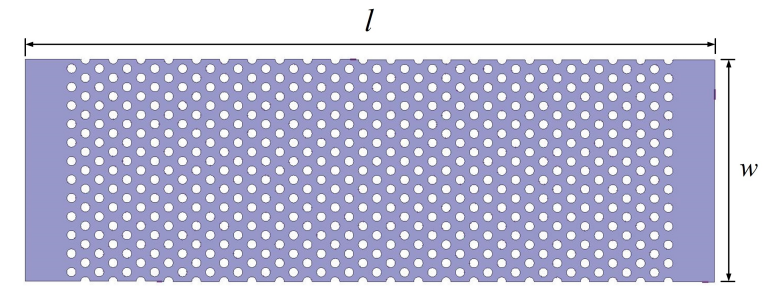

Fig. 1. The geometrical profile of the power plane

important significance of the RTC includes two folds: i) the new variable is strictly confined at the interfaces of neighboring subdomains; ii) only the normal component of the new variable is required while the $x, y$, and $z$-components need to be calculated in the traditional DG method. Thus, the number of DoFs in the proposed RTC-DG method is decreased remarkably.

\section{EleCtricAl-Thermal Co-SIMUlation}

To facilitate the electrical to the thermal coupling, the Joule heating resultant heat source $Q$ in (25) is obtained as

$$
Q=\mathbf{J} \cdot \mathbf{E}=\sigma|\nabla \Phi|^{2}
$$

To consider the impact of temperature rising on the material properties, the resistivity (denoted as $\rho$ with $\rho=\frac{1}{\sigma}$ ) of conductors is considered as a function of temperature $T$, which is updated in terms of

$$
\rho(T)=\rho_{0}\left[1+\alpha\left(T-T_{0}\right)\right]
$$

with $\rho_{0}$ indicating the initial value at ambient temperature $T_{0}$, and $\alpha$ designating the temperature coefficient of the conductors.

Due to the resulted electrical-thermal coupling matrix system is nonlinear, to efficiently solve it, the fixed point method is employed. The details of the solving scheme is presented as below: 


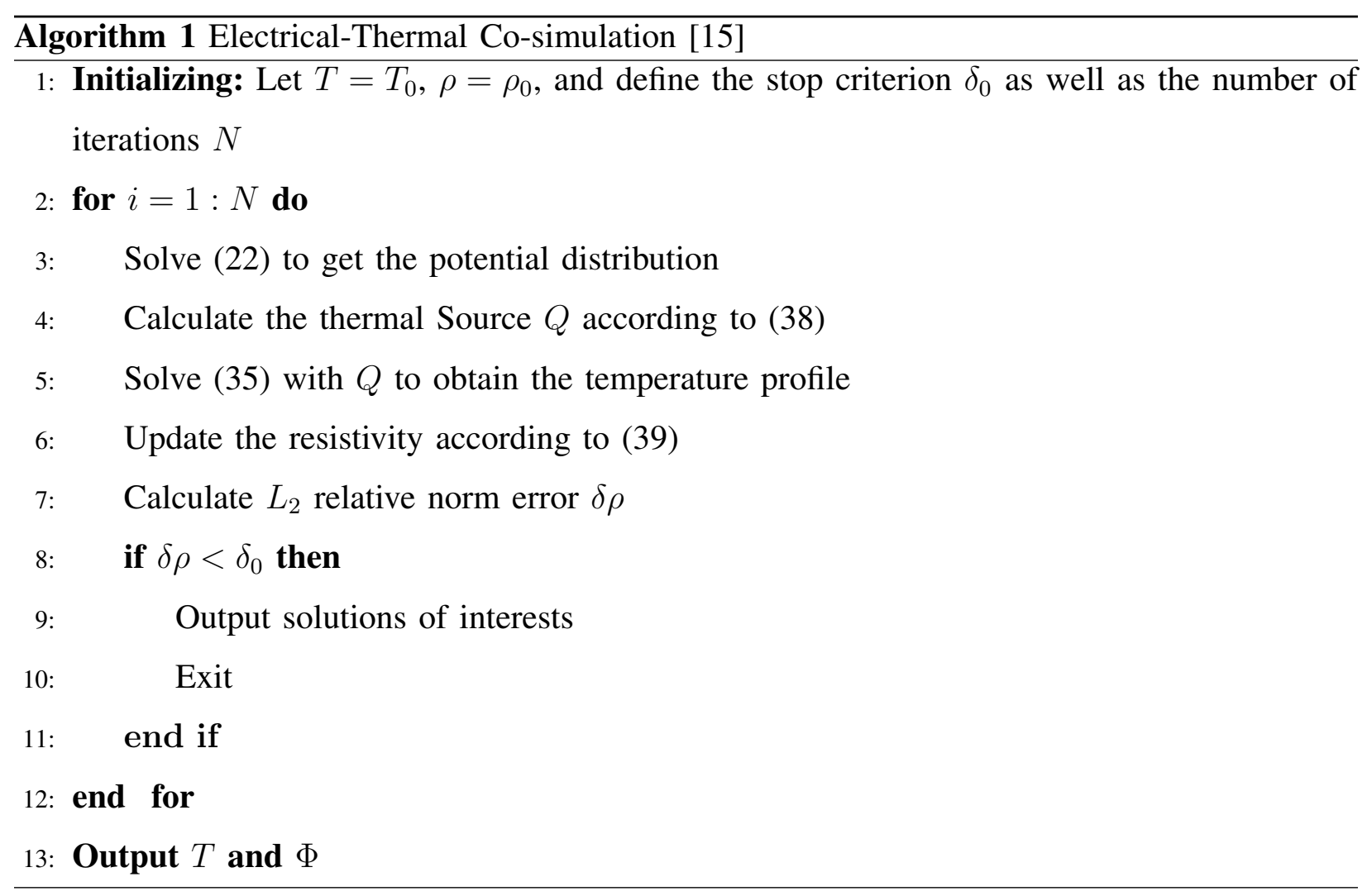

\section{Solving Strategy}

To solve the matrix equations in (22) and (35), in this work, a block-diagonal preconditioner is applied in advance. Let's take the electrical problem for illustration due to limited space. After applying the preconditioner, (22) is reformulated as

$$
\left[\begin{array}{cccc}
\mathbf{I} & \mathbf{M}_{1}^{-1} \mathbf{C}_{e}^{12} & \cdots & \mathbf{M}_{1}^{-1} \mathbf{C}_{e}^{1 K} \\
\mathbf{M}_{2}^{-1} \mathbf{C}_{e}^{21} & \mathbf{I} & \cdots & \mathbf{M}_{2}^{-1} \mathbf{C}_{e}^{2 K} \\
\vdots & \vdots & \ddots & \vdots \\
\mathbf{M}_{K}^{-1} \mathbf{C}_{e}^{K 1} & \mathbf{M}_{K}^{-1} \mathbf{C}_{e}^{K 2} & \cdots & \mathbf{I}
\end{array}\right]\left[\begin{array}{c}
\boldsymbol{v}_{e}^{1} \\
\boldsymbol{v}_{e}^{2} \\
\vdots \\
\boldsymbol{v}_{e}^{K}
\end{array}\right]=\left[\begin{array}{c}
\mathbf{M}_{1}^{-1} \tilde{\boldsymbol{f}}_{e}^{1} \\
\mathbf{M}_{2}^{-1} \tilde{\boldsymbol{f}}_{e}^{2} \\
\vdots \\
\mathbf{M}_{K}^{-1} \tilde{\boldsymbol{f}}_{e}^{K}
\end{array}\right]
$$

Then, (40) is going to be solved in a procedure similar to that employed in FETI method [16] in this work. The whole solution process involves two steps: i) the unknowns at the interfaces of subdomains are calculated firstly; ii) the interior unknowns in each subdomain are evaluated on the basis of solutions obtained in the first step.

To facilitate the solving procedure, the unknowns pertinent to subdomain $\Omega_{k}$ are divided into three groups: $\gamma_{k}^{I}$ for the expansion coefficients of the potential inside the subdomain; $\gamma_{k}^{s}$ for the expansion coefficients of the potential on the subdomain interface and $\lambda_{k}^{s}$ for the expansion 


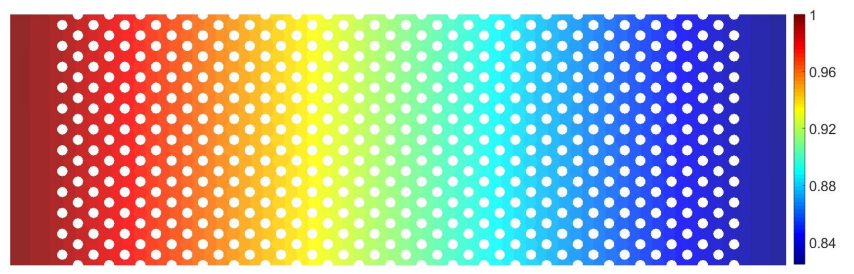

(a)

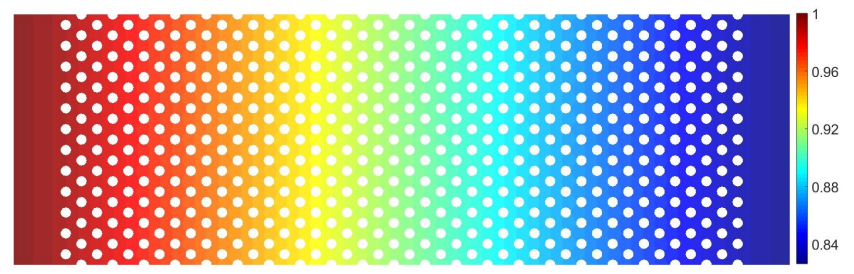

(b)

Fig. 2. The voltage distribution (unit: V) of the power plane. (a) Results calculated by DG method. (b) Results calculated by FEM.

coefficients of the current density on the subdomain interface, then the solution vector $\boldsymbol{v}_{e}^{k}$ can be rewritten as

$$
\boldsymbol{v}_{e}^{k}=\left[\begin{array}{c}
\gamma_{k}^{I} \\
\gamma_{k}^{s} \\
\lambda_{k}^{s}
\end{array}\right]
$$

Similarly, (23) and (24) could be rewritten as

$$
\mathbf{M}_{e}^{k}=\left[\begin{array}{ccc}
\mathbf{M}_{k}^{I I} & \mathbf{M}_{k}^{I S} & \mathbf{0} \\
\mathbf{M}_{k}^{S I} & \mathbf{M}_{k}^{S S} & \mathbf{M}_{k}^{S F} \\
\mathbf{0} & \mathbf{M}_{k}^{F S} & \mathbf{M}_{k}^{F F}
\end{array}\right]
$$

and

$$
\mathbf{C}_{e}^{k l}=\left[\begin{array}{ccc}
\mathbf{0} & \mathbf{0} & \mathbf{0} \\
\mathbf{0} & \mathbf{C}_{k l}^{S S} & \mathbf{C}_{k l}^{S F} \\
\mathbf{0} & \mathbf{C}_{k l}^{F S} & \mathbf{C}_{k l}^{F F}
\end{array}\right]
$$

By introducing a restriction operator $\mathbf{R}_{k}$, the surface unknowns $\overline{\boldsymbol{v}}_{e}^{k}$ can be conveniently extracted from $\boldsymbol{v}_{e}^{k}$, that is, 


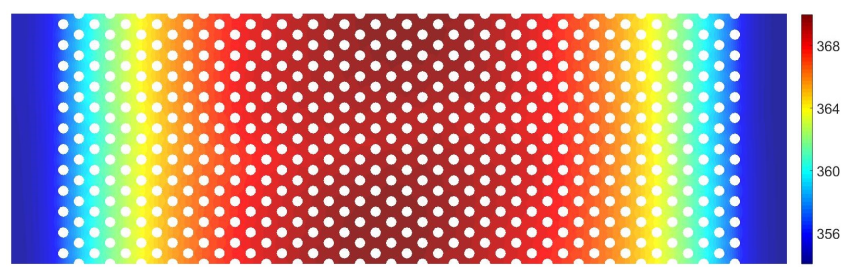

(a)

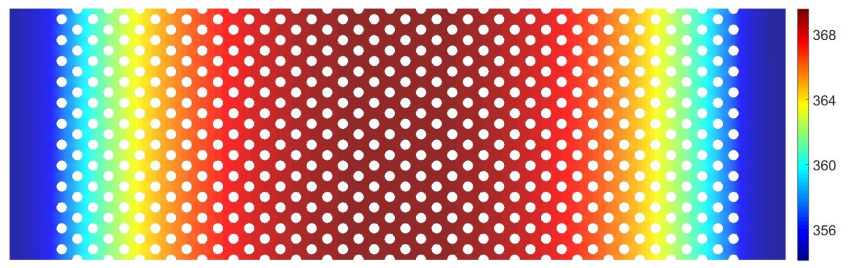

(b)

Fig. 3. The temperature distribution (unit: V) of the power plane. (a) Results calculated by DG method. (b) Results calculated by FEM.

$$
\overline{\boldsymbol{v}}_{e}^{k}=\mathbf{R}_{k} \boldsymbol{v}_{e}^{k}=\left[\begin{array}{c}
\gamma_{k}^{s} \\
\lambda_{k}^{s}
\end{array}\right]
$$

with

$$
\mathbf{R}_{k}=\left[\begin{array}{ccc}
\mathbf{0} & \mathbf{I}_{k}^{\phi} & \mathbf{0} \\
\mathbf{0} & \mathbf{0} & \mathbf{I}_{k}^{J}
\end{array}\right]
$$

Then, it can be easily shown that

$$
\mathbf{M}_{k}^{-1} \mathbf{C}_{e}^{k l} \boldsymbol{v}_{e}^{l}=\mathbf{M}_{k}^{-1}\left(\mathbf{C}_{e}^{k l} \mathbf{R}_{k}^{T} \mathbf{R}_{k}\right) \boldsymbol{v}_{e}^{l}=\mathbf{M}_{k}^{-1} \mathbf{C}_{e}^{k l} \mathbf{R}_{k}^{T} \overline{\boldsymbol{v}}_{e}^{l}
$$

with with the identity defined as,

$$
\mathbf{R}_{k}^{T} \mathbf{R}_{k}=\left[\begin{array}{ccc}
\mathbf{0} & \mathbf{0} & \mathbf{0} \\
\mathbf{0} & \mathbf{I}_{k}^{\phi} & \mathbf{0} \\
\mathbf{0} & \mathbf{0} & \mathbf{I}_{k}^{J}
\end{array}\right]
$$

With the relation in (46), a new matrix equation only containing the unknowns at the interfaces of neighboring subdomains can be obtained as

$$
\left[\begin{array}{cccc}
\mathbf{I} & \mathbf{Z}_{1} \overline{\mathbf{C}}_{e}^{12} & \cdots & \mathbf{Z}_{1} \overline{\mathbf{C}}_{e}^{1 K} \\
\mathbf{Z}_{2} \overline{\mathbf{C}}_{e}^{21} & \mathbf{I} & \cdots & \mathbf{Z}_{2} \overline{\mathbf{C}}_{e}^{2 K} \\
\vdots & \vdots & \ddots & \vdots \\
\mathbf{Z}_{K} \overline{\mathbf{C}}_{e}^{K 1} & \mathbf{Z}_{K} \overline{\mathbf{C}}_{e}^{K 2} & \cdots & \mathbf{I}
\end{array}\right]\left[\begin{array}{c}
\overline{\boldsymbol{v}}_{e}^{1} \\
\overline{\boldsymbol{v}}_{e}^{2} \\
\vdots \\
\overline{\boldsymbol{v}}_{e}^{K}
\end{array}\right]=\left[\begin{array}{c}
\overline{\boldsymbol{f}}_{e}^{1} \\
\overline{\boldsymbol{f}}_{e}^{2} \\
\vdots \\
\overline{\boldsymbol{f}}_{e}^{K}
\end{array}\right]
$$




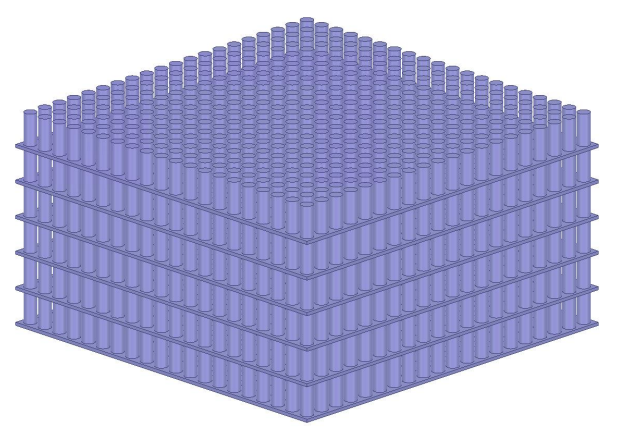

Fig. 4. The geometrical profile of the six-layered TSV array

TABLE II

Number of DoFs For $\Phi$ And J With $N_{\Phi}, N_{\mathrm{J}}, N_{T}$, AND $N_{\mathrm{q}}$ DENoting the number of DoFs For the Potential $\Phi$, THE CURRENT DENSITYJ, THE TEMPERATURE $T$, AND THERMAL FLUX q, RESPECTIVELY.

\begin{tabular}{|c|c|c|c|c|}
\hline Method & $N_{\Phi}$ & $N_{\mathrm{J}}$ & $N_{T}$ & $N_{\mathrm{q}}$ \\
\hline RTC-DG & 612,471 & 62,753 & 612,603 & 62,753 \\
\hline DG [15] & 612,471 & $1,837,413$ & 612,603 & $1,837,809$ \\
\hline
\end{tabular}

where

$$
\begin{aligned}
\mathbf{Z}_{k} & =\mathbf{R}_{k} \mathbf{M}_{k}^{-1} \mathbf{R}_{k}^{T} \\
\overline{\mathbf{C}}_{e}^{k l} & =\mathbf{R}_{k} \mathbf{C}_{k l} \mathbf{R}_{l}^{T} \\
\overline{\boldsymbol{f}}_{e}^{k} & =\mathbf{M}_{k}^{-1} \tilde{\boldsymbol{f}}_{e}^{k}
\end{aligned}
$$

Due to the number of the surface unknowns is much smaller than the total number of unknowns, thus a direct solver can be applied. In this work, the PARDISO solver is employed. Moreover, it is obviously noted that each row of the matrix (48) is independent to each other, thus they can be calculated in parallel to improve the calculation speed.

Once the surface unknowns $\overline{\boldsymbol{v}}_{e}^{k}(k=1,2, \cdots, K)$ are solved, the unknowns inside each subdomain can be computed via

$$
\boldsymbol{v}_{e}^{k}=\mathbf{M}_{k}^{-1}\left(\tilde{\boldsymbol{f}}_{e}^{k}-\sum_{l \neq k} \mathbf{C}_{k l} \mathbf{R}_{l}^{T} \overline{\boldsymbol{v}}_{e}^{l}\right)
$$

The calculation of each subdomain is completely independent to each other as well. Thus, it can be implemented in parallel to further reduce calculation time.

For the steady-state thermal issue, a same solving procedure is applied in this work. 


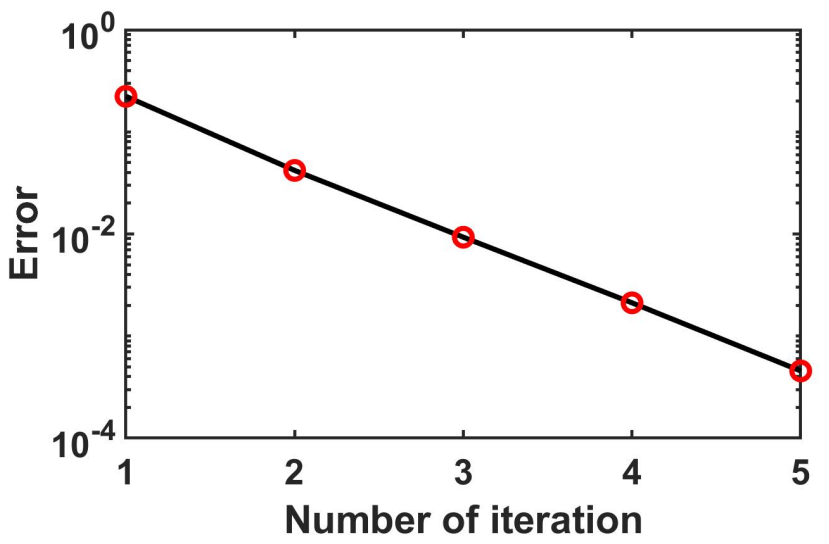

Fig. 5. The convergence property of the iterative electrical-thermal co-analysis scheme for the second example.

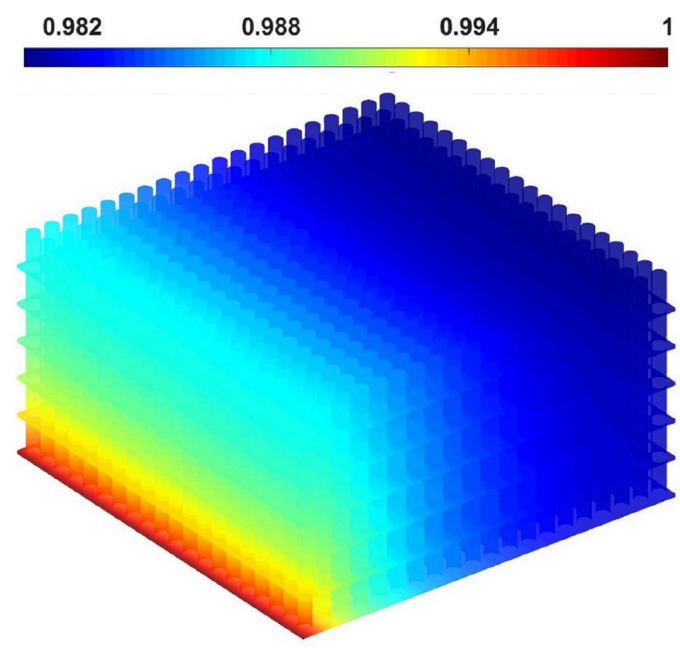

(a)

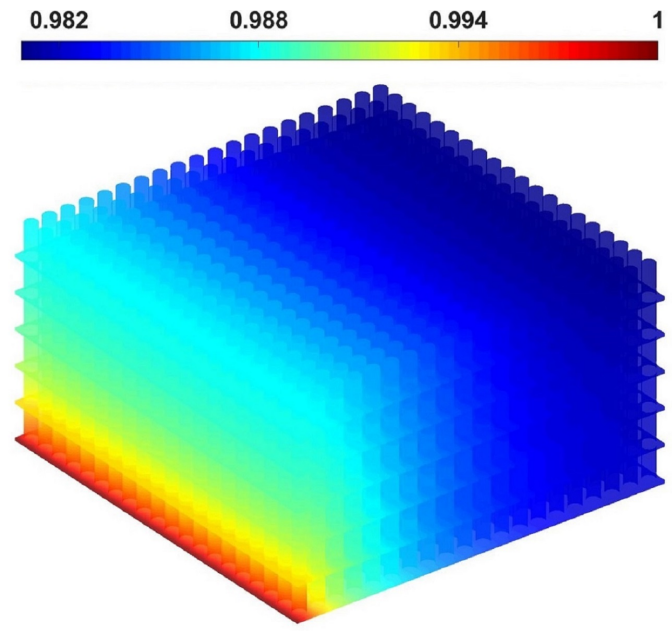

(b)

Fig. 6. The temperature distribution (unit: V) of the TSV array. (a) Results calculated by DG method. (b) Results calculated by FEM.

\section{NUMERICAL RESULTS}

In this section, to show the accuracy of the proposed RTC-DG method as well as the fast calculation property of the direct solver, several representative examples are investigated. For domain decomposition, a open source code METIS [19] is used to automatically regroup the generated tetrahedron mesh elements as evenly as possible, which leads to balance loads among subdomains. The computation of all examples is performed on the Intel Xeon Gold 6240R 2.4 $\mathrm{GHz}$ workstation with 48 cores. For electrical-thermal cosimulation, the stop criterion of the electrical-thermal solver is predefined as $\delta_{\rho}=0.001$. 


\section{A. A Power Plane with Swiss Cheese Effect}

To imitate the "Swiss Cheese Effects", a large number of holes are formed on a power plane shown in Fig. 1. The length $l$, the width $w$, and the thickness $t$ are $29.8 \mathrm{~mm}, 9.6 \mathrm{~mm}$, and $0.05 \mathrm{~mm}$, respectively. The radius of the via holes is $0.2 \mathrm{~mm}$, and the separation distances between two adjacent via holes are $0.4 \mathrm{~mm}$ along the width direction and $0.6 \mathrm{~mm}$ along the length direction, respectively.

To investigate the "Swiss Cheese Effects" resulted excessive Joule heating, a $1 \mathrm{~V}$ voltage source is imposed on the left end surface of the power plane and a resistive load with $R_{L}=0.01 \Omega$ is placed on the right end surface. The convection boundary condition is applied on the top and the bottom surface with $h=500 \mathrm{~W} /\left(\mathrm{m}^{2} \cdot \mathrm{K}\right)$. Other associated parameters are defined as $\rho_{0}=1.75 e-8 \Omega \cdot \mathrm{m}, \alpha=0.0039 \mathrm{~K}^{-1}, T_{a}=300 \mathrm{~K}$ and $\kappa=400 \mathrm{~W} /(\mathrm{m} \cdot \mathrm{K})$. The whole power plane is decomposed into 120 subdomains, and totally 167,559 tetrahedrons are involved. In table I, the number of DoFs for the associate variables are presented. For comparison, their counterparts in the traditional DG method are also given.

The electrical-thermal solver converges after 4 iterations, which is performed with 6 threads and takes around 52 seconds with peak memory cost about $3.8 \mathrm{G}$. The obtained voltage and temperature distributions are presented in Fig. 2 and 3. For accuracy validation, those obtained by FEM are also provided. Apparently, they agree with each other perfectly. Furthermore, the voltage drop with thermal effects is $0.1754 \mathrm{~V}$ while it's $0.1465 \mathrm{~V}$ without thermal effects, i.e., $19.8 \%$ more loss is caused owing to the Joule heating.

\section{B. A Multilayered PDN Chained by Through Silicon Via (TSV) Array}

In the second example, a PDN composed of six-layered copper planes is studied, where all power planes are vertically stacked via the TSV array. As shown in Fig. 4, the copper-filled TSV has radius $0.1 \mathrm{~mm}$ and height $0.5 \mathrm{~mm}$. The length, the width, and the thickness of each copper plane are $6.0 \mathrm{~mm}, 6.0 \mathrm{~mm}$ and $0.05 \mathrm{~mm}$, respectively. The separation between two adjacent TSVs is $0.3 \mathrm{~mm}$ along both $x$ - and $y$-axis. The electrical and thermal properties of the material are same as those in the first example. A voltage source $V_{D D}=1 \mathrm{~V}$ is placed over the left surface of the first layer, and the vias on the top layer are terminated by resistive loads with $R_{L}=1 \Omega$. The convection cooling boundary condition with $h=500 \mathrm{~W} / \mathrm{m}^{2} \mathrm{~K}$ is enforced at the bottom surface of each layer. 


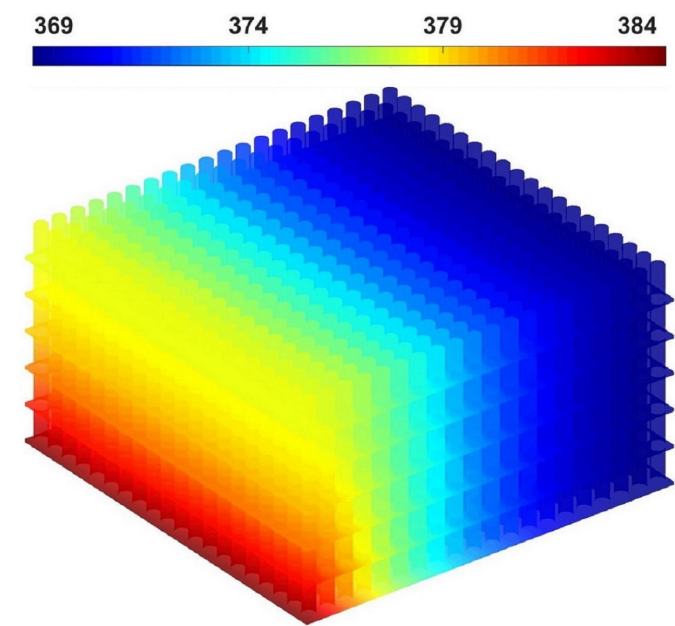

(a)

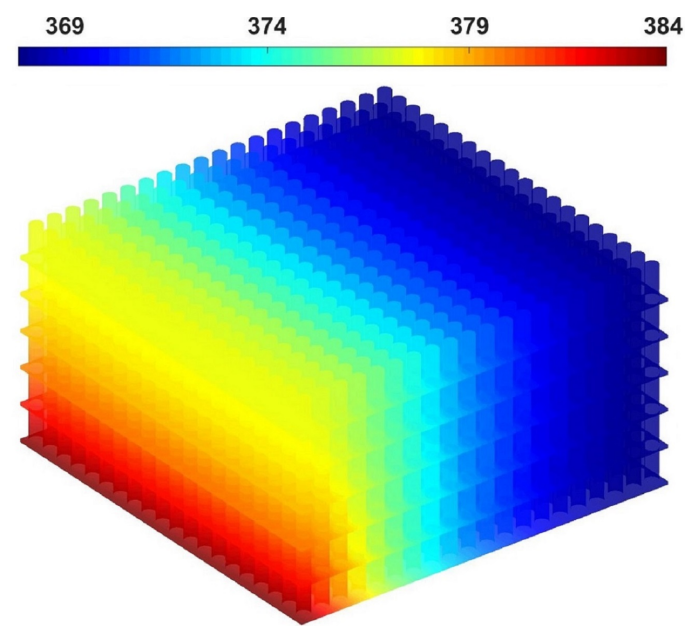

(b)

Fig. 7. The temperature distribution (unit: V) of the TSV array. (a) Results calculated by DG method. (b) Results calculated by FEM.

The whole structure is decomposed into 140 subdomains and totally 2, 164, 329 tetrahedrons are generated. The number of DoFs for $\Phi, \mathbf{J}, T$, and $\mathbf{q}$ are listed in Table II. As can be seen, for the auxiliary variable $\mathbf{J}$, the proposed RTC-DG reduces the number of DoFs from 1,837, 413 to 62,573 ; while for the auxiliary variable q, the number of DoFs is decreased from 1,837, 809 to 62,573 if the RTC-DG method is used. Thus, the RTC-DG method truly reduces the number of unknowns significantly.

The convergence rate of the electrical-thermal cosimulation is plotted in Fig. 5, only 5 iterations are required to reach the desired accuracy. In Fig. 6 and 7, the calculated voltage and the temperature distribution are presented. For reference, the results by FEM are given as well. Great agreements are observed. The maximum voltage drop with thermal effect is $19.5 \mathrm{mV}$, while the maximum voltage drop without considering Joule heating is $15.3 \mathrm{mV}, 27.4 \%$ more voltage drop is introduced owing to thermal effect and the lowest and the highest temperature of the whole structure are increased to $384.7 \mathrm{~K}$ and $368.5 \mathrm{~K}$, respectively.

For this example, the corresponding CPU time and the peak memory cost are about 48 minutes and $32 \mathrm{G}$, respectively. To have a better insight into the impact of the number of subdomains on the computation performance, the CPU time and peak memory cost versus the number of subdomains are shown in in Fig. 8 (a) and (b). In addition, the CPU time versus the number of threads pertinent to 140 subdomains is also provided in Fig. 8 (c). 


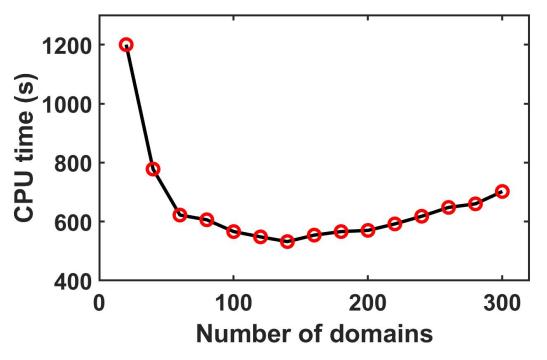

(a)

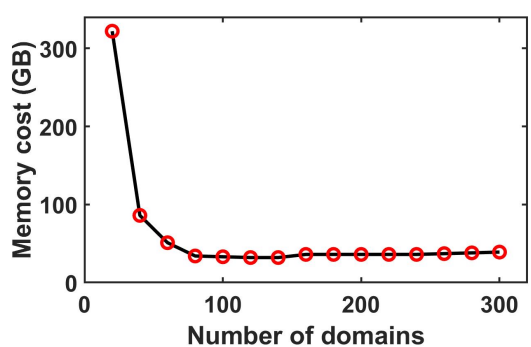

(b)

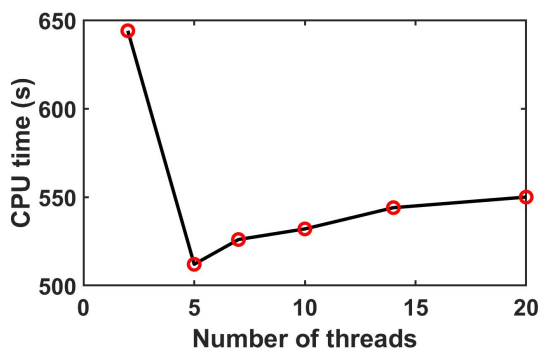

(c)

Fig. 8. (a) The CPU time versus the number of subdomains; (b) The peak memory cost versus the number of subdomains; (c) The CPU time versus the number of threads for 140 subdomains.

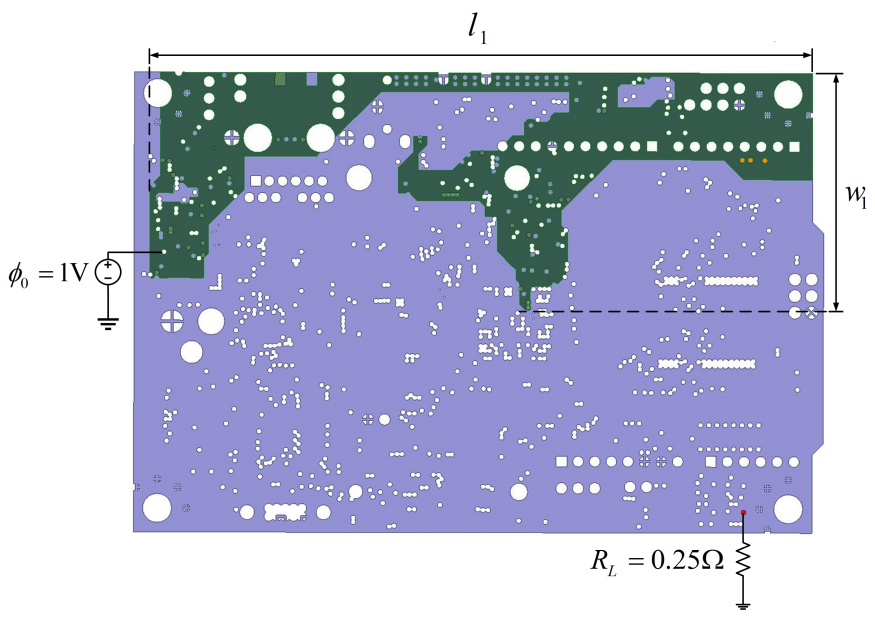

Fig. 9. The geometrical profile of the PCB with via-holes

C. A Two-layered Printed Circuit Board (PCB)

As the last example, a PCB composed of two power layers is studied. As shown in Fig. 9, the two power layers are connected by the three orange vias with radius and height respectively equal to $0.38105 \mathrm{~mm}$ and $1.77 \mathrm{~mm}$. The geometry parameters of the first layer are $l_{1}=101.422 \mathrm{~mm}$, 
TABLE III

Number of DoFs for $\Phi$ And J With $N_{\Phi}, N_{\mathrm{J}}, N_{T}$, ANd $N_{\mathrm{q}}$ DENoting The number of DoFs For the Potential $\Phi$, THE CURRENT DENSITYJ, THE TEMPERATURE $T$, AND THERMAL FLUX q, RESPECTIVELY.

\begin{tabular}{|c|c|c|c|c|}
\hline Method & $N_{\Phi}$ & $N_{\mathrm{J}}$ & $N_{T}$ & $N_{\mathrm{q}}$ \\
\hline RTC-DG & 260,466 & 20,591 & 260,512 & 20,591 \\
\hline DG [15] & 260,466 & 781,398 & 260,512 & 781,536 \\
\hline
\end{tabular}

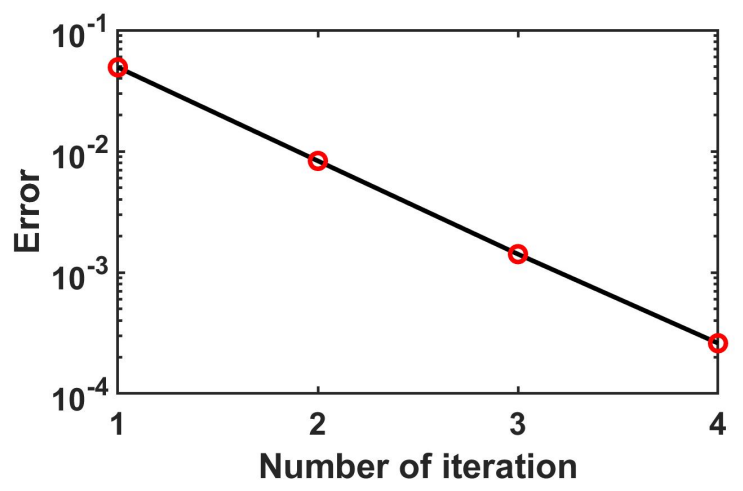

Fig. 10. The convergence property of the iterative electrical-thermal co-analysis scheme for the last example.

$w_{1}=36.297 \mathrm{~mm}$, and the thickness $t_{1}=0.03302 \mathrm{~mm}$, while the geometry parameters of the second layer are given as $l_{2}=103.886 \mathrm{~mm}, w_{2}=70.409 \mathrm{~mm}$, and $t_{2}=0.03302 \mathrm{~mm}$.

The voltage source $V_{D D}=1 \mathrm{~V}$ is placed on the first layer and a $0.25 \Omega$ resistive load is attached on a $0.38105 \mathrm{~mm}$ circular patch located over the second layer. The air convection cooling boundary condition with $h=10 \mathrm{~W} /\left(\mathrm{m}^{2} \cdot \mathrm{K}\right)$ is applied on the top surface of power planes. The entire structure is decomposed into 150 subdomains, and totally 686, 037 tetrahedrons are resulted. In Table III, the number of DoFs for the associated variables are provided.

The electrical-thermal cosimulation takes 4 iterations to reach the convergence requirement, where the convergence scheme presented in Fig. 10. In Fig. 11 and Fig. 12, the calculated voltage distribution and the temperature distribution are presented. It is noted that the temperature of the middle part of the first layer is higher than others owing to the "Swiss cheese" effect, which is increased to about $390 \mathrm{~K}$. The maximum voltage drop with the thermal effect is $0.1 \mathrm{~V}$, while the maximum voltage drop without the thermal effect is $0.084 \mathrm{~V}, 19.9 \%$ more voltage loss is caused due to the thermal effect. The calculation is performed with 6 threads, and the corresponding CPU time and the memory cost are around 7 minutes and $5 \mathrm{G}$, respectively. 


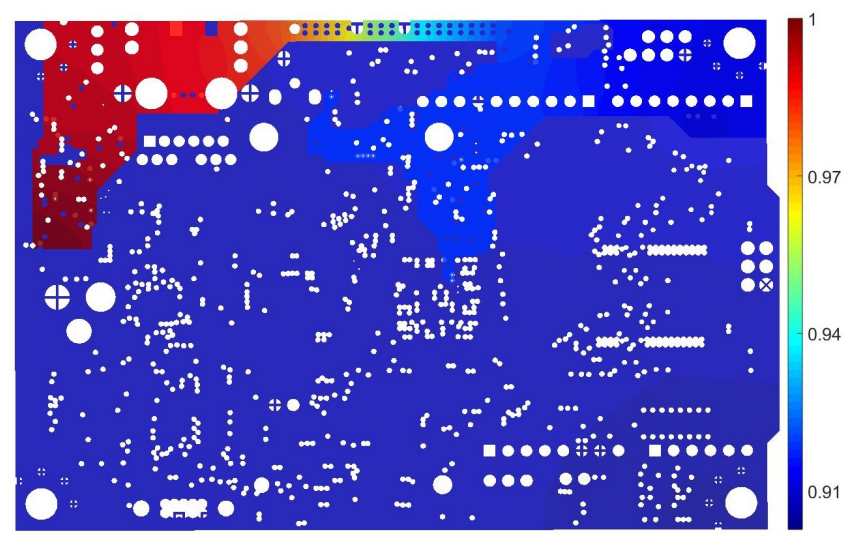

(a)

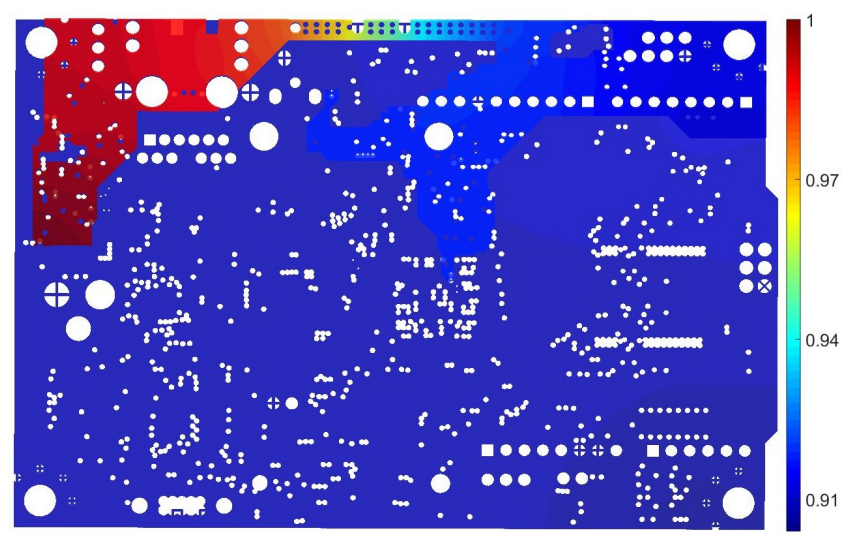

(b)

Fig. 11. The voltage distribution (unit: V) of the PCB. (a) Results calculated by DG method. (b) Results calculated by FEM.

\section{CONCLUSION}

A novel RTC-DG method is proposed for the DC IR-drop analysis of PDNs with thermal effects included. The proposed DG method directly discretizes the second-order spatial PDEs, and the introduced Robin Transmission Condition decreases the unknowns on the interface between subdomain than the traditional DG method. To make full use of the advantage of DG method in DDM, a FETI-like method is employed to solve the whole system in a subdomainby-subdomain scheme direct solver is resorted. The calculation is implemented in a parallel way, thus significantly improved the computational efficiency. The proposed approach is benchmarked by three examples by comparing with FEM. 


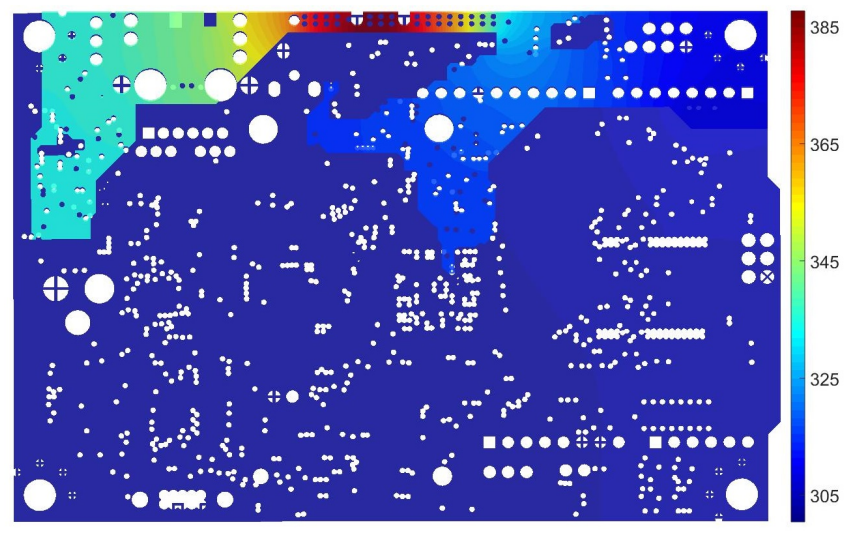

(a)

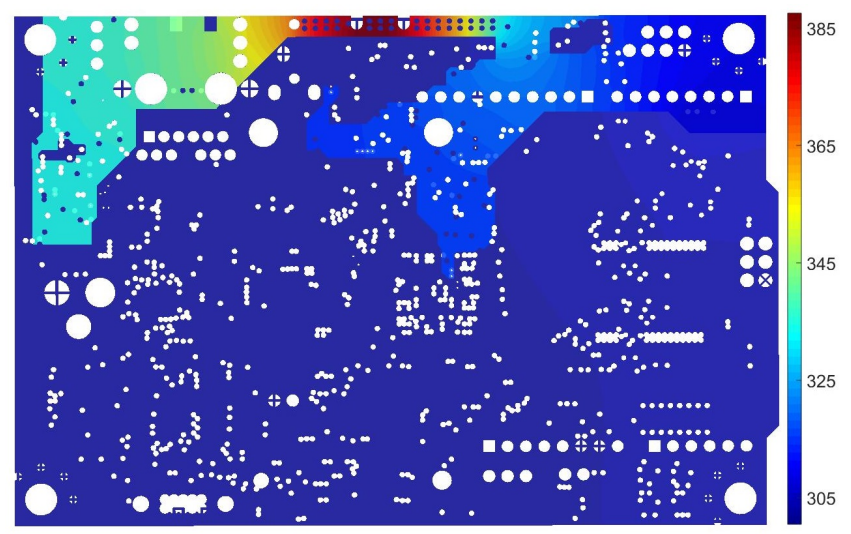

(b)

Fig. 12. The temperature distribution (unit: V) of the PCB. (a) Results calculated by DG method. (b) Results calculated by FEM.

\section{REFERENCES}

[1] M. S. Zhang and J. Fan, "IR-drop modeling and reduction for high performance printed circuit boards," IEEE Electromagn. Mag., vol. 4, no. 4, pp. 90-101, 2015.

[2] T.-L. Wu, F. Buesink, and F. Canavero, "Overview of signal integrity and EMC design technologies on PCB: fundamentals and latest progress," IEEE Trans. Electromagn. Compat., vol. 55, no. 4, pp. 624-638, Aug. 2013.

[3] D. Klokotov, J. Schutt-Aine, W. Beyene, D. Mullen, M. Li, R. Schmitt, and L. Yang, "Application of the latency insertion method to electrothermal circuit analysis," in Proc. IEEE 20th Conf. EPEPS, pp. 263-266, Oct. 2011.

[4] Y. Zhong and M. D. F. Wong, "Thermal-aware IR drop analysis in large power grid," in Proc. 9th ISQED, pp. 194-199, Mar. 2008.

[5] Y. Shao, Z. Peng, and J.-F. Lee, "Thermal-aware DC IR-drop coanalysis using non-conformal domain decomposition methods," Proc. Roy. Soc. A, Math., Phys. Eng. Sci., vol. 468, no. 2142, pp. 1652-1675, Jun. 2012.

[6] T. Lu and J. M. Jin, "Transient electrical-thermal analysis of 3-D power distribution network with FETI-enabled parallel computing,” IEEE Trans. Compon., Packag., Manuf. Technol., vol. 4, no. 10, pp. 1684-1695, Oct. 2014. 
[7] T. J. Lu and J. M. Jin, "Electrical-thermal co-cimulation for DC IR-drop analysis of large-scale power delivery," IEEE Trans. Compon., Packag., Manuf. Technol., vo. 4, no. 2, pp. 323-331, Feb. 2014.

[8] J. Xie and M. Swaminathan, "Electrical-thermal co-simulation of 3D integrated systems with micro-fluidic cooling and Joule heating effects,” IEEE Trans. Compon., Packag. Manuf. Technol., vol. 1, no. 2, pp. 234C246, Feb. 2011.

[9] P. Li and L. J. Jiang, "Integration of arbitrary lumped multiport circuit networks into discontinuous Galerkin time-domain analysis," IEEE Trans. Microw. Theory and Techn., vol. 61, no. 7, pp. 2525-2534, Jul. 2013.

[10] P. Li, Y. Shi, L. J. Jiang, and H. Bagci, "A hybrid time-domain discontinuous galerkin-boundary integral method for electromagnetic scattering analysis," IEEE Trans. Antennas Propag., vol. 62, no. 5, pp. 2841-2846, May 2014.

[11] J. M. Jin, The Finite Element Method in Electromagnetics, 2nd ed. New York, NY, USA: Wiley, 2003.

[12] C. Fletcher, Computational Techniques for Fluid Dynamics. Berlin, Germany: Springer-Verlag, 1991.

[13] P. Li, Y. L. Dong, M. Tang, J. F. Mao, L. J. Jiang, and H. Bagci, “Transient thermal analysis of 3-D integrated circuits packages by the DGTD method," IEEE Trans. Compon., Packag., Manuf. Technol., vol. 7, no. 6, pp. 862-871, Jun. 2017.

[14] Y. L. Dong, M. Tang, P. Li, and J. F. Mao, "Transient electromagnetic-thermal simulation of dispersive media using DGTD method," IEEE Trans. Electromagn. Compat., vol. 61, no. 4, pp. 1305-1313, Aug. 2019.

[15] P. Li, M. Tang, Z. X. Huang, L. J. Jiang and H. Bagci, “ DC IR-drop analysis of multilayered power distribution network by discontinuous Galerkin method with thermal effects incorporated," IEEE Trans. Compon., Packag. Manuf. Technol., vol. 10, no. 6, pp. 1035-1042, June 2020.

[16] Y. Shao, Z. Peng, K. H. Lim, and J.-F. Lee, "Non-conformal domain decomposition methods for time-harmonic maxwell equations," Proceedings of the Royal Society A: Mathematical, Physical and Engineering Sciences, vol. 468, pp. 2433-2460, 2012.

[17] M. Bollhofer, A. Eftekhari, S. Scheidegger, and O. Schenk, "Large-Scale Sparse Inverse Covariance Matrix Estimation", SIAM J. Sci. Comput., vol. 40., no.1, pp. A380-A401, 2019.

[18] P. Castillo, B. Cockburn, I. Perugia, D. Schotzau, "An a priori error analysis of the local discontinuous Galerkin method for elliptic problems," SIAM, vol. 38, no. 5, pp. 1676-1706, 2000.

[19] George Karypis and Vipin Kumar, "Multilevel k-way partitioning scheme for irregular graphs," J. Parallel Distrib. Comput., vol. 48, no. 1, pp. 96-129, 1998.

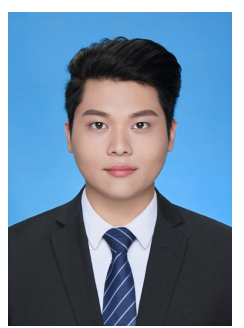

An Fa Yang was born in Lu'an, Anhui, China, in 1997. He received the B. S. degree in communication engineering from Shanghai University, Shanghai, in 2019.

He is currently pursuing the Master Degree with Shanghai Jiao Tong University, Shanghai, China. His research interests include multiscale and multiphysics computational algorithms, and parallel computing. 
Min Tang (Member, IEEE) was born in 1980. He received the B.S. degree in electronic engineering from Northwestern Polytechnical University, Xi'an, China, in 2001, the M.S. degree in electrical engineering from Xi'an Jiao Tong University, Xi'an, China, in 2004, and the Ph.D. degree in electronic engineering from Shanghai Jiao Tong University, Shanghai, China, in 2007.

Since 2007, he has been a Faculty Member in Shanghai Jiao Tong University, where he is currently a Professor in the Department of Electronic Engineering. He was a Postdoctoral Research Fellow at the University of Hong Kong, Hong Kong, from 2010 to 2012. His research interests include signal and power integrity of high-speed circuits, multiscale and multiphysics modeling of integrated systems.

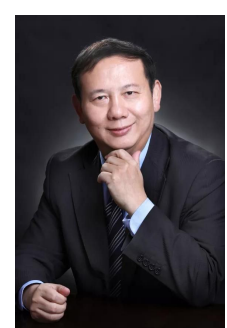

Jun Fa Mao (Fellow, IEEE) was born in 1965. He received the B.S. degree in radiation physics from the University of Science and Technology of National Defense, Changsha, China, in 1985, the M.S. degree in experimental nuclear physics from the Shanghai Institute of Nuclear Research, Shanghai, China, in 1988, and the Ph.D. degree in electronic engineering from Shanghai Jiao Tong University, Shanghai, in 1992.

He was a Visiting Scholar with The Chinese University of Hong Kong, Hong Kong, from 1994 to 1995, and a Post-Doctoral Researcher with the University of California at Berkeley, Berkeley, CA, USA, from 1995 to 1996. Since 1992, he has been a Faculty Member with Shanghai Jiao Tong University, where he is currently a Chair Professor and the Dean of the School of Electronic Information and Electrical Engineering. He is member of the Chinese Academy of Science, Beijing. He has authored or coauthored more than 200 journal papers (including more than 120 IEEE journal papers) and 150 international conference papers. His research interests include the interconnect and package problem of integrated circuits and systems, and analysis and design of microwave circuits.

Dr. Mao was a member of 2012šC2014 IEEE MTT-S Fellow Evaluation Committee and a member of 2015 IEEE Fellow Committee. He received the National Natural Science Award of China in 2004, the National Technology Invention Award of China in 2008, and the National Science and Technology Advancement Award of China in 2012. He is a Chief Scientist of the National Basic Research Program of China, a Project Leader of the National Science Foundation for Creative Research Groups of China, a Cheung Kong Scholar of the Ministry of Education, China, the Director of the Microwave Society of China Institute of Electronics, and the 2009šC2015 Chair of the IEEE MTT-S Shanghai Chapter. He was the Founder and the $2007 \check{\mathrm{S}} 2009$ Chair of the IEEE Shanghai Section. 


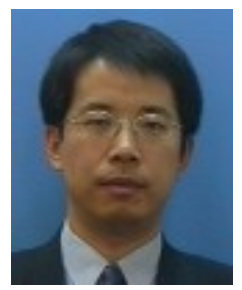

Li Jun Jiang (Fellow, IEEE) received the B.S. degree in electrical engineering from the Beijing University of Aeronautics and Astronautics, Beijing, China, in 1993, the M.S. degree from Tsinghua University, Beijing, in 1996, and the Ph.D. degree from the University of Illinois at UrbanašCChampaign, Champaign, IL, USA, in 2004.

From 1996 to 1999, he was an Application Engineer with Hewlett-Packard Company, Beijing. He served as a Scientific Consultant with Hong Kong Applied Science and Technology Research Institute Company Ltd., Hong Kong, from 2010 to 2011. He was with Semiconductor Research Cooperation (SRC) Industrial Liaison, Hong Kong, for several academic projects. Since 2004, he has been a Post-Doctoral Researcher, a Research Staff Member, and a Senior Engineer with the IBM T. J. Watson Research Center, Yorktown Heights, NY, USA. Since 2009, he has also been an Associate Professor with the Department of Electrical and Electronic Engineering, The University of Hong Kong, Hong Kong. Since 2013, he has also been a Senior Visiting Professor with Tsinghua University, Beijing. He has been serving as a Panelist on the Expert Review Panel for the Hong Kong Research and Development Center for Logistics and Supply Chain Management Enabling Technologies, Hong Kong, since 2013. He has been involved collaboratively with many international researchers.

Dr. Jiang is a member of the IEEE Antennas and Propagation Society (AP-S), the IEEE Microwave Theory and Techniques Society (MTT-S), the IEEE Electromagnetic Compatibility Society (EMC-S), the ACES, and the Chinese Computational Electromagnetics Society. He has been an elected TPC Member of the IEEE Electrical Design of Advanced Packaging and Systems Symposium (EDAPS) since 2010 and the IEEE Electrical Performance of Electronic Packaging since 2014. He was a TPC Member of the 2013 IEEE International Conference on Microwave Technology and Computational Electromagnetics. He has been a TC-9 Member and a TC-10 Member of the IEEE Electromagnetic Compatibility Society since 2011. He was a Scientific Committee Member of the 2010 IEEE Simulation and Modeling of Emerging Electronics, the Special Session Organizer of the IEEE EDAPS, the IEEE Electromagnetic Compatibility, Applied Computational Electromagnetics Society, AsiašCPacific Radio Science Conference, Progress in Electromagnetics Research Symposium, and a Co-Organizer of the HKU Computational Science and Engineering Workshops from 2010 to 2012. He was a recipient of the IEEE MTT Graduate Fellowship Award in 2003 and the Y. T. Lo Outstanding Research Award in 2004. He was a Session Chair of many international conferences. He was the TPC Chair of the 7th International Conference on Nanophotonics/the 3rd Conference on Advances in Optoelectronics and Micro/Nano Optics, the TPC Co-Chair of the 12th International Workshop on Finite Elements for Microwave Engineering and the 2013 International Workshop on Pulsed Electromagnetic Field at Delft, The Netherlands, and the General Chair of the 2014 IEEE 14th HK AP/MTT Postgraduate Conference. He was an Associate Guest Editor of the Proceedings of the IEEE special issue from 2011 to 2012. He is an Associate Editor of the IEEE TRANSACTIONS ON ANTENNAS AND PROPAGATION and Progress in Electromagnetics Research. He also serves as a reviewer for the IEEE TRANSACTIONS on several topics and other primary electromagnetics and microwave-related journals. 


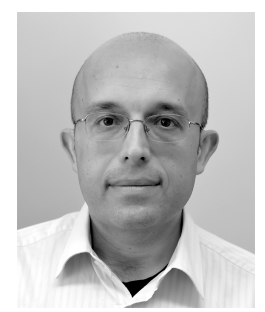

Hakan Bagci (Senior Member, IEEE) received the B.S. degree in Electrical and Electronics Engineering from the Bilkent University, Ankara, Turkey, in June 2001 and the M.S. and Ph.D. degrees in Electrical and Computer Engineering from the University of Illinois at Urbana-Champaign (UIUC), Urbana, in August 2003 and January 2007, respectively. From June 1999 to July 2001, he worked as an Undergraduate Researcher at the Computational Electromagnetics Group, Bilkent University. From August 2001 to December 2006, he was a Research Assistant at the Center for Computational Electromagnetics and Electromagnetics Laboratory, UIUC. From January 2007 to August 2009, he worked as a Research Fellow at the Radiation Laboratory, University of Michigan. In August 2009, he joined the Division of Physical Sciences and Engineering at the King Abdullah University of Science and Technology (KAUST) as Assistant Professor of Electrical Engineering. His research interests include various aspects of computational electromagnetics with emphasis on time-domain integral equations and their fast marching-on-in-time-based solutions, well-conditioned integral-equation formulations, and development of fast hybrid methods for analyzing statistical EMC/EMI phenomena on complex and fully loaded platforms.

Dr. Bagci was the recipient of the 2008 International Union of Radio Scientists (URSI) Young Scientist Award and the 20042005 Interdisciplinary Graduate Fellowship from the Computational Science and Engineering Department, UIUC. His paper titled "Fast and rigorous analysis of EMC/EMI phenomena on electrically large and complex structures loaded with coaxial cables" was one of the three finalists (with honorable mention) for the 2008 Richard B. Schulz Best Transactions Paper Award given by the IEEE Electromagnetic Compatibility Society. He authored and co-authored eight finalist papers in the student paper competitions at the 2005, 2008, and 2010 IEEE Antennas and Propagation Society International Symposiums and 2013 and 2014 Applied Computational Electromagnetics Society Conference.

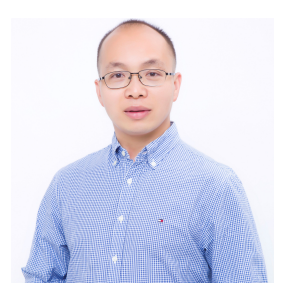

Ping Li (Senior Member, IEEE) received the B.S. degree in Physical Electronics Engineering from the University of Electronic Science and Technology of China (UESTC), in June 2008 and the Ph.D. degrees in Electrical and Electronic Engineering from the University of Hong Kong (HKU), Hong Kong, in August 2014. From September 2008 to August 2010, he was a master student of UESTC. From October 2014 to December 2015, he was a Postdoctoral Fellow at the Computational Electromagnetics Lab, King Abdullah University of Science and Technology (KAUST). From December 2015 to December 2016, he was a Postdoctoral Fellow at the On-Chip Electromagnetics Lab, Purdue University. From January 2017 to August 2018 , he joined the Department of Electrical and Electronic Engineering at HKU as a Research Assistant Professor. From September 2018 to August 2019, he was a research scientist at KAUST as well as a Honorary Assistant Professor at HKU. Since August 2019, he joined Shanghai Jiao Tong University as an Associate Professor.

Dr. Li was the recipient of the 2018 Joint IEEE EMC and APEMC Outstanding Young Scientist Award, 2018 ACES-China Young Scientist Award, and 40th PIERS Young Scientist Award in Japan. He was the recipient of the Okawa Research Foundation Grant of Japan. Besides, he was the recipient of 2018 Best Annual Paper Prize on Chinese Journal of Radio Science, and the recipient of Second Prize Award for Natural Sciences of Chinese Institute of Electronics, 2019. Besides, his paper was selected as the Finalist paper in 29th International Review of Progress in Applied Computational Electromagnetics and 2014 International Symposium on Electromagnetic Compatibility, and he won the Best Student Paper Award in 12th International Workshop on Finite Elements for Microwave Engineering. 